\title{
Publisher's Note: Spatio-orientational decoherence of nanoparticles [Phys. Rev. A 94, 033828 (2016)]
}

Benjamin A. Stickler, Birthe Papendell, and Klaus Hornberger (Received 18 July 2017; published 26 July 2017)

DOI: 10.1103/PhysRevA.96.019903

This paper was published online on 16 September 2016 with errors in Eqs. (38) and (39) and an error in the text before Eq. (39). Equation (38) should read as

$$
D_{\mathrm{RS}}=\frac{\gamma_{0}|b|^{2} \hbar^{2}}{3}\left[\left(\frac{\Delta \chi}{\chi_{\|}}\right)^{2}+\frac{(k \ell)^{2}}{30} \frac{\Delta \chi \chi \perp}{\chi_{\|}^{2}}+\frac{7(k \ell)^{4}}{1800}\left(\frac{\chi \perp}{\chi_{\|}}\right)^{2}\right]
$$

Equation (39) should read as

$$
D_{\mathrm{BB}}=40 \frac{c\left(V_{0} \chi_{\|} \hbar\right)^{2}}{\pi^{3}}\left(\frac{k_{\mathrm{B}} T}{\hbar c}\right)^{7}\left[\frac{\Delta \chi^{2}}{\chi \|} \zeta(7)+\frac{28}{15} \frac{\Delta \chi \chi \perp}{\chi_{\|}^{2}}\left(\frac{k_{\mathrm{B}} T \ell}{\hbar c}\right)^{2} \zeta(9)+\frac{98}{5}\left(\frac{\chi \perp}{\chi \|}\right)^{2}\left(\frac{k_{\mathrm{B}} T \ell}{\hbar c}\right)^{4} \zeta(11)\right] .
$$

The text before Eq. (39) should read as "follows from Planck's law, $\mu(k)=k^{2} / n_{\mathrm{g}} \pi^{2}\left[\exp \left(\hbar c k / k_{\mathrm{B}} T\right)-1\right]$ with temperature $T$." The equations and text have been corrected as of 18 July 2017. The equations and text are incorrect in the printed version of the journal. 